\title{
The effects of expectancy on corticospinal excitability: passively preparing to observe a movement
}

\author{
Pablo Arias, ${ }^{1}$ Verónica Robles-García, ${ }^{1}$ Nelson Espinosa, ${ }^{1}$ Yoanna Corral- \\ Bergantiños, ${ }^{1}$ Laura Mordillo-Mateos, ${ }^{2}$ Kenneth Grieve, ${ }^{3}$ Antonio Oliviero, ${ }^{2}$ and Javier \\ Cudeiro $^{1}$
}

\author{
${ }^{1}$ Laboratory of Neuroscience and Motor Control (NEUROcom), Department of Medicine-INEF-Galicia and Institute \\ of Biomedical Research of Coruña, University of A Coruña, A Coruña, Spain; \\ ${ }^{2}$ Functional Exploration and Neuromodulation of Nervous System Investigation Group, Hospital Nacional de \\ Parapléjicos, Servicio de Salud de Castilla-La Mancha, Toledo, Spain; and \\ ${ }^{3}$ Faculty of Life Sciences, University of Manchester, Manchester, United Kingdom
}

\begin{abstract}
The corticospinal tract excitability is modulated when preparing movements. Earlier to movement execution, the excitability of the spinal cord increases waiting for supraspinal commands to release the movement. Movement execution and movement observation share processes within the motor system, although movement observation research has focused on processes later to movement onset. We used single and paired pulse transcranial magnetic stimulation on M1 $(n=12)$, and electrical cervicomedullary stimulation $(n=7)$, to understand the modulation of the corticospinal system during the "preparation" to observe a third person's movement. Subjects passively observed a hand that would remain still or make an index finger extension. The observer's corticospinal excitability rose when "expecting to see a movement" vs. when "expecting to see a still hand." The modulation took origin at a spinal level and not at the corticocortical networks explored. We conclude that expectancy of seeing movements increases the excitability of the spinal cord.
\end{abstract}

\section{Keywords}

Movement preparation; Movement observation; TMS; Cervicomedulary stimulation; Spinal excitability; Corticospinal excitability 
THE EXECUTION OF SIMPLE MOTOR TASK involves a stream of processes within our corticospinal (CS) system before, during and after movement. Movement preparation (MP) involves changes at both the cortical and spinal levels of the motor system, which have different temporal profiles, depending on whether the movement is self-generated or externally triggered, and its kinematic and kinetic profile (Chen et al. 1998; Conrad et al. 1983; Eichenberger and Ruegg 1984; Frank 1986; Riedo and Ruegg 1988).

In MP processes spinal excitability (SE) is modulated up to $3 \mathrm{~s}$ before movement onset (Duclos et al. 2008; Mellah et al. 1990; Requin et al. 1977), and later fine-tuning of corticospinal excitability (CSE) triggers the action. Reaction time (RT) protocols are basic paradigms in the study of MP. In a RT protocol, after a waiting period of rest, a preparatory signal starts the preparatory period (Mellah et al. 1990), also termed warning signal and foreperiod (Soto et al. 2010); this preparatory period varies in duration in different studies and extends to the moment of the response signal (Mellah et al. 1990) (also called imperative stimulus, go stimulus, etc.); the lag-time from the response signal to movement onset is called reaction-time period, and within this period is the premotor-time which lasts from response-signal to the onset of myoelectrical activity (Eichenberger and Ruegg 1984). In RT protocols there are changes in the CSE after the response-signal and before the motor response (RT period), separate from CSE changes in the foreperiod to the response-signal (this is the period from warning-signal to responsesignal) (Mellah et al. 1990). Thus, in RT, as in self-generated movement, there is a later phasic SE increase which advances movement EMG onset (Eichenberger and Ruegg 1984; Frank 1986; van Elswijk et al. 2007). Changes in CSE during MP are also focused upon the cortex, and there seems to be a period when the spinal cord is ready (Frank 1986), but the M1 output is inhibited (or lacks facilitation), as if inhibited until the proper time of movement execution (Aoki et al. 2002; Conrad et al. 1983; Davranche et al. 2007; Soto et al. 2010).

Movement observation (MO; i.e., a task where the observer watches movements made by another person, without observer execution) and movement execution share common processes, like modulation of the neuronal activity at different levels of the motor system (Bonnet et al. 1997; di Pellegrino et al. 1992; Jeannerod and Decety 1995), but the motor system involvement during MO has only been studied after movement onset (di Pellegrino et al. 1992), not at the preparation phase (i.e., preparation of the motor system to observe movements). It is of interest to understand if the motor system also prepares to observe a movement. This might improve our understanding of the physiological processes occurring in later phases of observation, when the movement is actually observed; during this phase there is a modulation in the CSE of the observer (Baldissera et al. 2001; Fadiga et al. 1995). It is, however, important to consider that a modulation of the CSE of the observer might also be present prior to the start of the expected movement; in such case the dynamics of the modulation present during MO, and its meaning, should take account of the changes in the CSE induced by movement expectation prior to actual observation of any movement.

Here we test a preliminary hypothesis to explain motor system preparation for MO. Since an increase in the SE before movement initiation has previously been reported (Frank 1986; Mellah et al. 1990), perhaps serving to prepare the CS tract prior to discharge at cortical command (Conrad et al. 1983; Mellah et al. 1990; Soto et al. 2010), we predict that, in a process involving the observation of movements, there will be an adaptation of the motor system of the observer prior to the onset of the movement observed; this adaptation will involve a spinal preparation for a possible motor response to the movement subsequently observed.

We evaluated CSE and SE by means of single and paired pulse transcranial magnetic stimulation (TMS) on the motor cortex, and electric cervicomedullary stimulation (CMS) in subjects passively observing a movie of a third person's hand which would remain still or make a movement (an index finger extension). We used TMS to evaluate the cortical excitability because it is able to activate the CS system transynaptically at cortical level, evoking multiple descending volleys (I-waves) originated at cortical level (Di Lazzaro et al. 1998). Contrarily, electric CMS activates the CS axons at brain stem level, and it is not influenced by changes of the cortical excitability (Ugawa et al. 1991). For this reason, electric CMS is suitable to evaluate the SE virtually at any spinal level. Subjects were made aware of whether the hand 
would move or would stay still, but, in the cases where movement was expected, the stimulus was delivered before movement was seen, during the movement preparatory phase.

\section{MATERIALS AND METHODS}

All experiments presented here were approved by the University of A Coruña Ethics Committee. Subject's consent was obtained according to the Declaration of Helsinki.

We performed two blocks of experiments. In experiment 1 we explored CSE and corticocortical excitability by means of single and paired-pulse TMS. In experiment 2 we explored SE by means of CMS.

\section{Experiment 1}

Subjects. Twelve healthy volunteers participated (4 men and 8 women, age range 24 to $37 \mathrm{yr}$ ). All were right-handed according to the Edinburgh Handedness Inventory (Oldfield 1971), with normal or corrected-to-normal vision and were medication-free in the week prior to testing. Subjects were seated comfortably on a chair with their left forearm resting on a table and both hands covered from view, while observing a PC monitor $1 \mathrm{~m}$ in front of them, which was used to display all the movies shown. The movies would show several repetitions of three different kinds of stimuli: 1) a black screen; 2) a movie showing hand which remains still throughout its presentation; and 3) a movie showing the same hand as before but executing a movement, an index finger extension (with return to the initial position) in a cycle lasting $720 \mathrm{~ms}$.

Preparation of the movie stimuli. All movies were prepared/assembled using Pinnacle System Software. Initially, the operators hand was filmed and a single slow finger movement recorded at 25 frames per second (fps).

From this 25 fps recording, 10 frames were selected to create the movie stimuli (Fig. 1): one Still frame (the last frame preceding index finger extension during recording), and nine "Movement" frames [frames showing finger position at maximum extension (MAX), 1/9 MAX and $\sim 2 / 9$ MAX $\ldots \sim 8 / 9$ MAX]. 


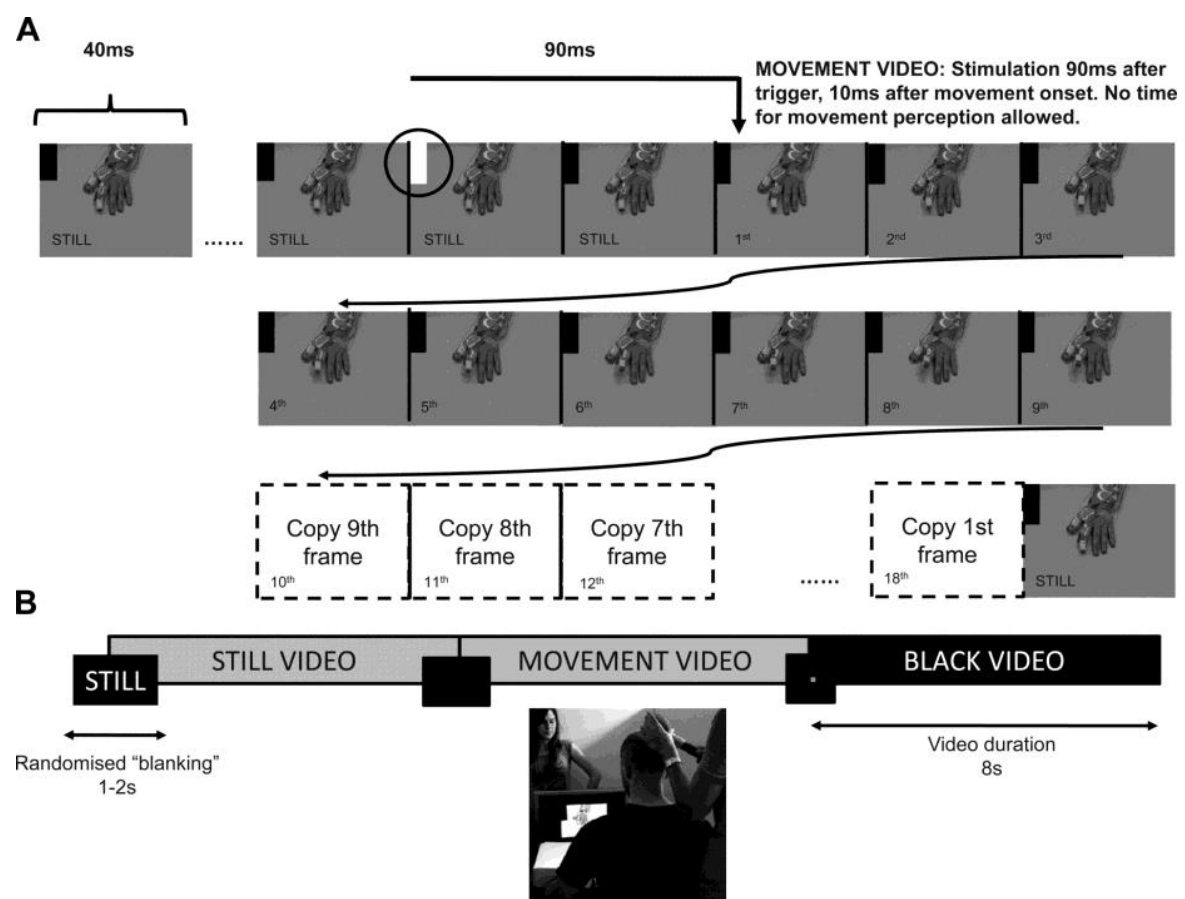

Fig. 1.Construction of the stimuli and the experimental paradigm. A: the final set of stimuli shown to the subject was a concatenation of several smaller movies, depicting either a single finger movement of 720-ms duration (movie video stimulus) within an 8-s movie, or an 8-s movie with the hand shown but no movement (still movie stimulus). The text identifying frames was not part of the actual stimulus. The black rectangle in the upper left corner of each frame was changed to white on the second frame before the movement began, but was not visible to the subject. It was used to activate a photocell which in turn triggered the transcranial magnetic stimulation (TMS) pulses. $B$ : a black masking frame covered a randomized period at the junctions of the short movies. Prior to every still video stimulus (except one per series, used as a test for maintained attention, see text) the word "Quieta" (STILL) was displayed in the middle of this black frame; this allowed preparation for movement observation if the word "Quieta" was absent. In some trials, the black masking included a $2 \times 2-\mathrm{cm}$ fixation gray square, shown for $2 \mathrm{~s}$; in such cases the following 8 $\mathrm{s}$ movie showed a black screen (BLACK). The lady shown facing the camera (absent during experimental sessions) is presented to make clear the size of the hand shown on the screen. The subject's hands were covered to avoid any possible visual interference with the hand on the screen.

For the experiments we created three movies: MOVE, STILL or BLACK.

To create the MOVE movie, frames were arranged in this order: $4 \mathrm{~s} \times$ Still frame + Movement frames (1/9 MAX - . . 7/9 MAX - 8/9 MAX - MAX - . . 1/9 MAX) + 3.3 s Still frame. The sequential progression of the "Movement" frames showing the position of the finger up to MAX and back created the movement stimulus.

The STILL movie was an 8-s presentation of only the Still frame (i.e., the same frame presented repeatedly).

The BLACK movie was an 8-s presentation of a black frame (see Fig. 1).

The final set of stimuli shown to the subject was a concatenation of several smaller movies, depicting either a single finger movement of 720-ms duration (MOVE) within an 8-s movie; an 8-s movie with the hand shown but no movement (STILL); or a neutral 8-s BLACK movie.

A Blank (black) screen of random duration of between 1 and $2 \mathrm{~s}$ overlaid part of the period "between" movies, thereby preventing prediction. To differentiate Blank screen separating movies from the BLACK movie, the latter were identified by a $2 \times 2$ gray square fixation spot, which appear for the first $2 \mathrm{~s}$ of the 
movie. STILL movies were identified by the Spanish word "Quieta" ("Still") displayed on screen during the preceding blank period and was only displayed prior to a STILL movie (Fig. 1B).

Movie stimuli characteristics. The overall stimuli presented to the subjects was a randomized sequence of 48 MOVE movies, 48 cued STILL movies, 48 BLACK movies and 2 uncued STILL movies (which the subjects were asked to verbally report, acting as a control/measure of attention; if the subject did not report the uncued STILL, data from the whole movie were discarded and repeated).

At some point during each of the movie stimulus (BLACK, STILL and MOVE), one stimulation pulse was delivered to the CS system of the observer (see below).

Brain stimulation. Each subject received 146 single TMS pulses (48 for each MOVE, STILL and BLACK movies, and 2 uncued STILL), delivered using two monophasic Magstim $200^{2}$ stimulators connected with a Bi-Stim module (Magstim, Whiteland, Dyfed, UK) and a 70-mm figure-of-eight coil. The coil was oriented tangentially to the skull, with the handle pointing $45^{\circ}$ back/downwards to induce currents in the postero-anterior direction. Test stimuli were set at an intensity of $125 \%$ rest motor threshold (RMT). The conditioning stimuli were set at 90\% active motor threshold (AMT) (Di Lazzaro et al. 1999; Ziemann et al. 1996). RMT and AMT were calculated on the hot spot of the first dorsal interosseous (FDI) on the right hemisphere, which was marked. RMT was the minimum intensity required to produce $50 \%$ of liminal responses $(\sim 50 \mu \mathrm{V})$ in 10 consecutive trials (Rossini et al. 1994), while looking at a black screen and the muscle at rest. For AMT, the muscle was slightly activated $(\sim 5-$ $10 \%$ of maximal voluntary contraction), and the liminal response required were about $200 \mu \mathrm{V}$ (Rossini et al. 1994). For the paired-pulse tests, a pair of TMS pulses was delivered such that the conditioning stimulus advanced the conditioned stimulus by $2-3 \mathrm{~ms}$ for assessing short intracortical inhibition (SICI), and by $10-15 \mathrm{~ms}$ for assessing intracortical facilitation (ICF).

Brain stimulation and movie presentation synchrony. Timing of TMS/CMS pulses relative to the observed movement was controlled by the addition of a $2 \times 5 \mathrm{~cm}$ white rectangle in the Still frame appearing $80 \mathrm{~ms}$ prior to movement onset (circled in Fig. 1), and at the same time in the STILL and BLACK movies. The spot activated a photocell which triggered the TMS/CMS stimulator, and this trigger was used to deliver test TMS/CMS pulses at a randomly selected point within a 3- to 4 -s timewindow after hand appearance on the screen (or after fixation extinguishing for BLACK). Furthermore, in MOVE condition, the movie triggered TMS/CMS at $10 \mathrm{~ms}$ after movement onset. While the forthcoming movement was cued at the start of the movie sequence by the absence of "Quieta," thereby allowing time for CS preparation, the 10-ms delay after movement onset does not allow any movement perception, since is not long enough for any significant visual processing (Maunsell et al. 1999).

Instructions given to the subjects. Subjects were required to view passively, paying attention to the movies, fixating on a probe attached to the tip of observed index finger, or to the fixation spot during the Blank movie sections. They were also instructed to be relaxed, but, if they wished to blink, to try to do so during black-transitional screens. They were told to verbally report the absence of movement if not cued as attentional checking (see above Movie stimuli characteristics section). 


\section{Experiment 2}

Seven healthy subjects participated ( 4 men and 3 women, age range 26-43 yr). All were right-handed. Experimental procedures were the same as experiment 1, except that subjects received one CMS for each movie: MOVE, STILL or BLACK. Due to the unpleasant/uncomfortable sensations induced by CMS, the number of movies was reduced to 31 (10 each of Movement, Still and Blank, with 1 uncued Still). CMS was applied with a Digitimer D180 stimulator connected to a pair of $\mathrm{Ag}-\mathrm{AgCl}$ electrodes. Electrodes were placed behind the mastoid processes, with the anode at the left and the cathode at the right. Stimulation intensity was increased progressively to the highest intensity acceptable to the subject which had a motorevoked potential (MEP) of the same latency as intensities evoking liminal responses, such that, as the induced MEP amplitude (MEPa) increased, there was no latency shift. The mean intensity used in the experiments was $600 \pm 33 \mathrm{~V}$ (SEM)

\section{Signal processing.}

MEPs (filtered between 3 and 3,000 Hz) were recorded over the left FDI, thumb opponent (THU) and extensor digitorum (EXT) muscles, using $\mathrm{Ag} / \mathrm{AgCl}$ surface electrodes and D360 amplifiers (Digitimer). We explored the effect on these three muscles because the FDI, the THU and the EXT are muscles with different roles in the movement to be observed (an index finger extension). During an index finger movement, apart from the main agonist discharge (EXT), there is coactivation in different hand muscles compensating joint torques induced by the action (Darling and Cole 1990). The FDI, with a role in the flexion of the index metacarpophalangeal joint, has insertions to the index and the thumb metacarpal bone (Hudson et al. 2009), which might be stabilized by different muscles, like the THU. The exploration of the three muscles allows us to study if the adaptations of the motor system to observation of a movement were specific to the role of the different muscles involved in the movement to be observed.

Recordings were sampled at $10 \mathrm{kHz}$ and stored via a CED1401 (CED). MEPa and the level of background EMG (Thompson et al. 1991) (area/time from $-80 \mathrm{~ms}$ to $-10 \mathrm{~ms}$ relative to the TMS/CMS pulse) were calculated with customized MatLab programs (The Mathworks). To control a potential effect of the EMG background (EMGbg) level on the MEPa, if the EMGbg difference between conditions was $P<0.100$ in a given muscle, such "preactivated" events were excluded (events with EMGbg exceeding the mean of the whole recording $+0.5 \mathrm{SD}$ ). Event exclusion was performed such that, if one muscle was preactivated in one event, the MEPa of the three muscles for that event was discarded, so that the remaining events included in the analysis had no significant differences in EMGbg between conditions $(P$ $>0.100)$. None of the events was discarded from the TMS protocols. During CMS, the final analysis included per subject a median of 4/10 events in BLACK, 5/10 in STILL and 5/10 in MOVE. CMS is very reliable with such amount of events (Martin et al. 2009).

\section{Statistical Analysis}

The analyzed variables were the same for both experiments: MEPa, $\mathrm{mV}$ (peak-to-peak size of the MEP for each delay, in each muscle); EMGbg, $\mathrm{mV}(\mathrm{mV} \times \mathrm{ms}$ normalized in time) for each delay, in each muscle.

MEPa (and EMGbg) were normalized in amplitude. Including all subjects in the TMS protocol, we calculated the average of the unconditioned (test) MEP for BLACK. This value was used as a normalizing factor, and therefore all values (test, SICI or ICF) for all conditions (BLACK, STILL and MOVE) were divided by it, for all subjects. Afterwards, for ICF and SICI, we calculated the conditioned MEP/unconditioned MEP. A similar procedure was repeated to normalize the CMS data (all unconditioned values). 
Analysis of variance (ANOVA) with repeated measures was used to understand how the variables were modulated by the preparation to see a movement. For ANOVA, univariate approaches were used, the Mauchly test was applied to check sphericity, and, if in violation, Greenhouse coefficients $(\varepsilon)$ were applied to correct the degrees of freedom. The ANOVA-repeated measures model contained two withinsubject factors. Factor expectancy had two levels (STILL and MOVE); muscle had three levels (FDI, THU, and EXT). For the analysis of the effect of the presence of a hand vs. a black screen, the model was the same but factor expectancy was termed as condition, and the two levels were STILL and BLACK. We also evaluated under the same model the difference between the levels BLACK and MOVE.

To compare the effects of the stimuli in the cortical or spinal component of the tract, a between-factor was included in the ANOVA model. Therefore, the ANOVA presents two within-subjects factors and also includes a between-subjects factor (which is the factor group). Factor group included two levels (TMS group and CMS group). This way we can evaluate if there are significantly different behaviors in the unconditioned values (single pulses) of the two groups, depending on expectancy and muscle.

For paired-pulse analysis, SICI and ICF were first analyzed separately as their physiological mechanisms differs. However, because a nonsignificant decrease in SICI in parallel with a nonsignificant increase in ICF might underlie a significant cortical excitability change, a subsequent analysis was performed, including an extra factor in the ANOVA mentioned above, factor SICI-IFC. Normality of distributions was checked by means of a Kolgomorov-Smirnov test for one sample. Significance was set at $P \leq 0.05$; values represented in graphs are mean and standard error of mean (SEM).

\section{RESULTS}

Modulation of CSE in Absence of Movement Expectation: BLACK vs. STILL

Figure $2 A$ shows that CSE is reduced when comparing STILL to BLACK. This effect was significant $[F(1,11)=9.275 P=0.011]$ and was not different for each of the three muscles studied $[F(2,22)=1.344$ $P=0.281]$. The ratio of MEPa values (conditioned $\mathrm{MEPa}$ /unconditioned MEPa) were used to calculate SICI (conditioning to conditioned interpulse interval 2-3 ms), and ICF (interpulse interval 10-15 ms). Paired-pulse analysis showed that this effect was not explained by changes in corticocortical circuits $[F(1,11)=0.060 P=0.811]$, and the absence of effect was not different for SICI or IFC in any of the muscles $[F(2,22)=1.043, P=0.369]$ (Fig. $2 B$ ). Here, the excitability of the spinal cord appeared responsible for this effect (Fig. $2 C$, overlaid traces shown in $2 D$ ); as the effect on the MEP induced by CMS was not significantly different from the behavior induced by TMS in any of the muscles $[F(2,34)=$ $0.024, P=0.975]$, while the decreased in excitability was highly significant $[F(1,17)=11.053, P=$ 0.004]. This observation suggests that sight of the hand object, coupled with the knowledge that no movement is expected, is enough to modulate the excitability of the spinal cord. 

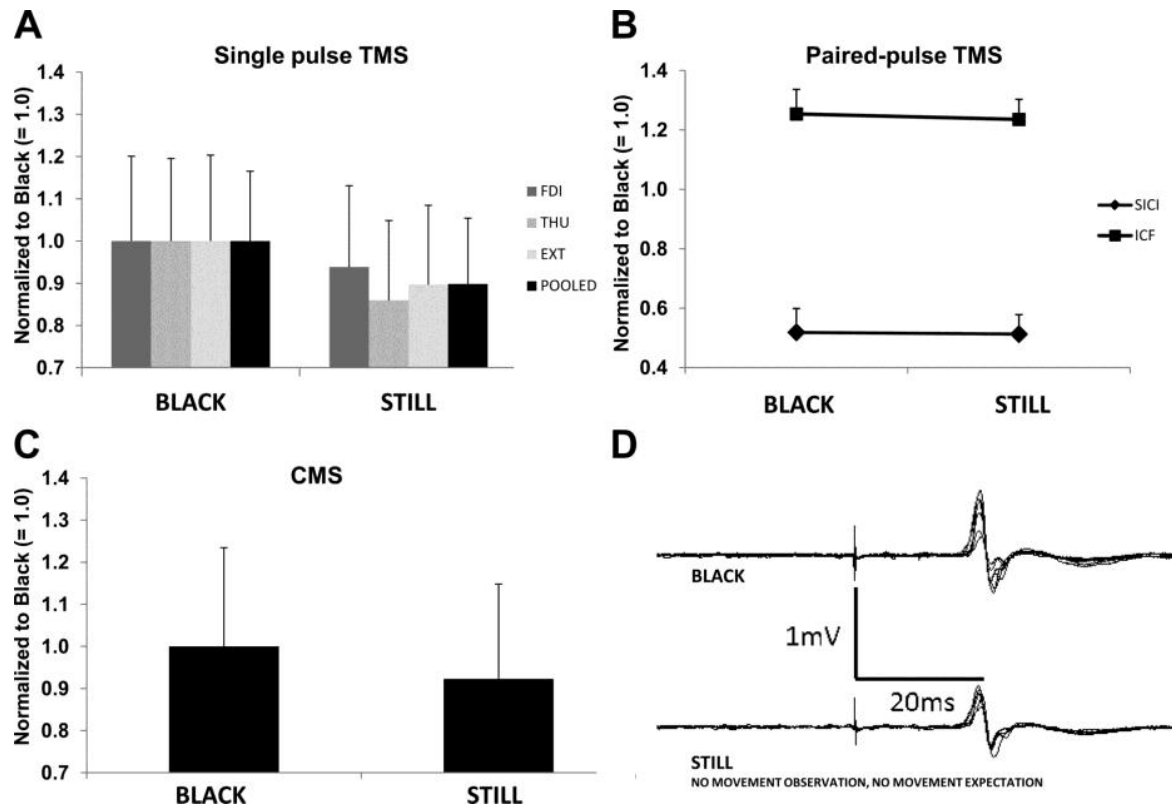

D

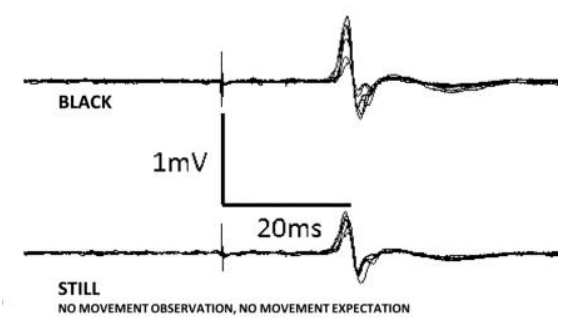

Fig. 2.Cortical and spinal excitability modulation by a stationary hand vs. absence of movement effector (BLACK). A: corticospinal excitability (CSE) is reduced significantly when observing a hand which will not move (STILL) compared with a Blank screen (BLACK); the three muscles did not respond differently. $B$ : this effect is not explained by a modulation of corticocortical networks; neither short intracortical inhibition (SICI) nor intracortical facilitation (ICF) were changed if comparing conditions. The three muscles did not differ in response-profile, so that they are represented pooled. $C$ : the STILL condition induced a significant reduction in the spinal excitability with regards to the BLACK situation, with no different effect for the three muscles. $D$ : example traces of spinal modulation in the first dorsal interosseous using CMS, from an individual subject, when no stimulus was visible (BLACK), and when it was visible, but no movement expected (STILL).

\section{Corticospinal Preparation to Observe Movements}

MEPa values induced by single TMS pulses are illustrated in Fig. 3A, which shows that corticospinal tract excitability increases by about $10 \%$ when $\mathrm{MO}$ is expected, compared with when the hand is seen but no MO is expected $[F(1,11)=5.450, P=0.040$, "pooled" muscles, STILL vs MOVE]. This significant effect was observed individually in all three muscles evaluated (Fig. 3A; FDI, THU and EXT), and the responses of the individual muscles were not significantly different $[F(2,22)=0.140, P=0.870]$. EMGbg did not affect the results, as there was no significant difference between values in the pooled muscle value between expected MO and no expected MO $[F(1,11)=0.806 P=0.389$ ] [again individual muscle responses were not significantly different, $\left.F(2,22)_{\varepsilon=0.542}=0.341, P=0.588\right]$. While this single-pulse TMS study shows that motor system is facilitated when MO is expected, the experiment does not reveal the location of the facilitation (cortical or spinal). 


\section{A}

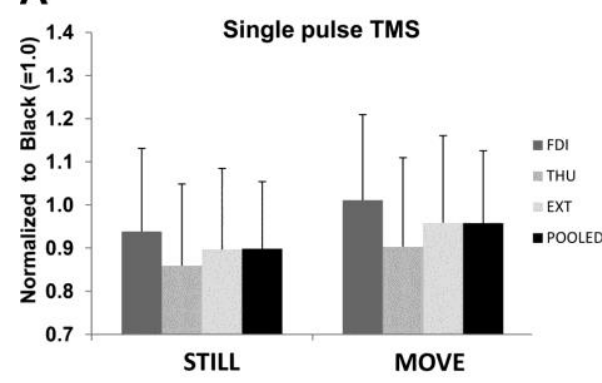

C

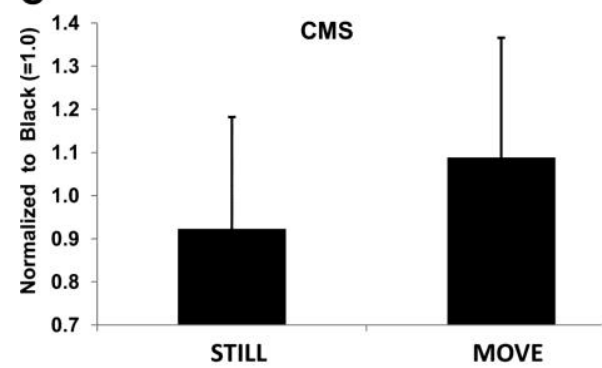

B

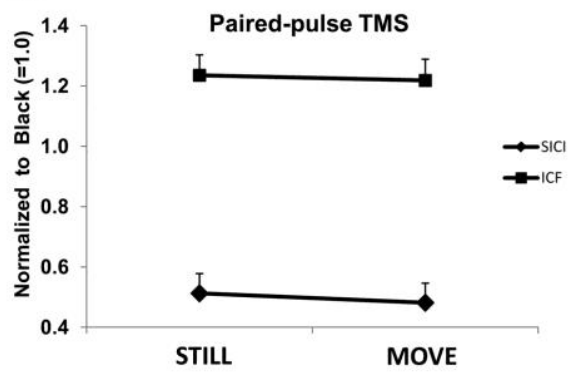

D

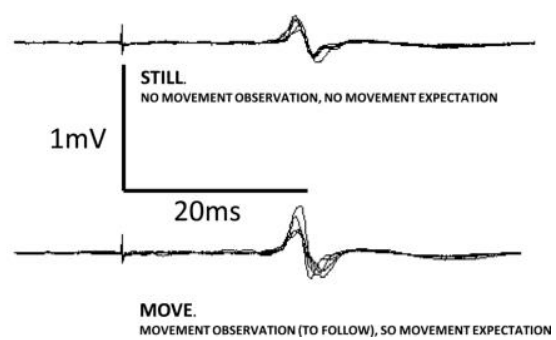

Fig. 3. Corticospinal modulation by movement observation expectancy. A: CSE was significantly increased comparing responses to the observation of a stationary hand which would make a movement (MOVE) vs. a hand which would remain stationary (STILL). This behavior, showing that the mere expectancy of the oncoming movement increases the CSE, was observed in all of the muscles evaluated, since the three muscles did not significantly differ in their responses. $B$ : neither SICI nor ICF was changed between the MOVE and STILL conditions, although SICI was significant inhibited and ICF significantly elevated in each case. Since the same pattern was observed in each of the three muscles, they were presented pooled. $C$ : spinal excitability showed a very significant increase when subjects knew they were observing a hand under the MOVE condition compared with STILL. Again, the pooled muscle data are plotted, as the three muscle responses were not significantly different. $D$ : example traces of spinal modulation in the first dorsal interosseous using CMS, from an individual subject, when no movement was expected (STILL), and when it was expected (MOVE).

\section{Corticocortical Preparation to Observe Movements}

Paired-pulse TMS responses are shown in Fig. 3B. The expected significant elevation in ICF and decrease in SICI were easily detected $[F(1,11)=62.725, P<0.001]$. More importantly, neither showed a significant difference between expected MO (MOVE) and no expected MO [STILL, $F(1,11)=0.663, P=$ $0.433]$. Figure $3 B$ shows pooled data, and again the individual muscle responses were not significantly different $[F(2,22)=0.223, P=0.802$, data not shown]. EMGbg did not affect the results. This pairedpulse TMS study shows that intracortical inhibitory and excitatory circuits (and the balance between them) are unchanged when MO is expected.

\section{Spinal Preparation to Observe Movements}

Following electric CMS, spinal motoneurons showed increased excitability when MO was expected, compared with when no MO was expected, and this was observed after excluding the events with higher EMGbg activity (a significant effect was also observed if including all events; data not reported). The MEPa values were $\sim 10 \%$ larger [Fig. $3 C$, MOVE vs. STILL, $F(1,6)=31.204, P=0.001$ ]. Overlaid representative single traces are illustrated for the two conditions in Fig. 3D. The increase was not significantly different for each of the three muscles $[F(2,12)=0.043, P=0.958]$. Again, this effect was observed without change in the level of EMGbg activity $[F(1,6)=3.515, P=0.110]$ between MOVE vs. STILL, and this was not different for the three muscles $[F(2,12)=0.432, P=0.493]$. 
The effect of CMS was similar in magnitude and direction to that observed following single TMS pulses when both were compared. The triple interaction, expectancy $\times$ muscle $\times$ group, showed that group's behaviors were not significantly different $[F(2,34)=0.013, P=0.987]$. Muscle-by-muscle profile did not differ with either level of expectancy $[F(2,34)=0.142, P=0.868$, expectancy $\times$ muscle $]$; and the excitability was higher in MOVE compared with STILL $[F(1,17)=31.016, P<0.001$, main effect of factor expectancy].

\section{Compound Effect Due to Observation and Expectation}

Thus far our results show a reduction in SE of the observer by the mere presence of a hand in view, and an increase in the SE if the movement is expected. Now, with the analysis of the BLACK vs. MOVE, reflecting the comparison of a neutral condition vs. a compound condition resulting from observing (hand) and expecting (movement), we show that the level of excitability is not significant different $[F(1,17)=0.601, P=0.449]$, and this behavior is not significantly different for the three muscles $[F(2,34)$ $=2.001, P=0.151]$, nor when the excitability is evaluated with TMS or CMS $[F(2,34)=0.063, P=$ $0.939]$.

The evaluation of the changes in the excitability of the corticocortical circuits explored with pairedpulse TMS did not explain any modulation between BLACK and MOVE, and the only main effect observed only proved that, in all cases, the response of ICF was significantly higher than that of SICI $[F(1,11)=69.754, P<0.001]$. The rest of main effects and interactions were never significant.

\section{DISCUSSION}

This work provides novel evidence that the excitability of motor CS networks, as tested by TMS and CMS in humans, can be increased by the expectancy of MO. Since CMS activates the CS axons directly, at cervicomedullary junction (Ugawa et al. 1991), it is a useful tool to evaluate a change in excitability of the corticospinal pathways at the spinal cord level. CMS reflects the intrinsic and extrinsically modulated excitability properties of spinal motoneurons and of CS axons at a long distance from the soma and the axon hillock of the cortical motoneurons (Ugawa et al. 1991). On the other hand, TMS activates CS pathways trans-synaptically, so it is useful to study motor cortex excitability (Hallett 2000).

We have shown that the expectancy of MO increases the amplitude of EMG responses evoked either by TMS or CMS. Given the above, we suggest that the similar effect of expectancy of MO on EMG responses, evoked by both TMS and CMS, indicates that the increase in excitability takes place at the level of circuits within the spinal cord; however, the presence of spinal facilitation cannot rule out small changes in M1 cortical excitability measured using single-pulse TMS. As far as paired-pulses are concerned, we cannot rule out completely a cortical involvement mediated by circuits not explored by the SICI and ICF protocols.

SICI as a response to paired-pulse TMS is believed to reflect the excitability of inhibitory cortical circuits mediated by GABAa receptors, and expectancy of MO had no effects on SICI. ICF as a response to paired-pulse TMS seems to depend on the activity of intracortical excitatory circuits (Di Lazzaro et al. 2003; Ziemann et al. 1998). Again expectancy of MO had no effects on the facilitatory mechanisms tested by ICF. Therefore, it appears that corticocortical networks explored by SICI and ICF are uninvolved in the preparation of the motor system to see a movement. However, it is important to consider that weak modulations of corticocortical circuits during the preparation to observe movements might be overwhelmed by the larger excitability changes taking place at the spinal cord level. It is possible that other TMS protocols based on paired-pulse on M1, evaluating short-ICF (Ziemann et al. 1998), or long intracortical inhibition (Valls-Sole et al. 1992), on triple-pulse on M1, evaluating late cortical desinhibition (Cash et al. 2010), or other neurophysiological techniques (e.g., EEG, MEG, etc.) may be able to detect cortical involvement of M1 or other higher order cortical motor areas in the presence of spinal cord facilitation. 
To summarize, the expectancy of MO increases the amplitude of EMG responses evoked by TMS and CMS, but no effects on inhibitory and facilitatory intracortical circuits were observed. We conclude that the increase in excitability takes place at spinal cord level, and that a cortical involvement is less likely.

Our motor systems are involved during both MO and movement itself, sharing some processes (Bonnet et al. 1997; Jeannerod and Decety 1995), for instance the motor system resonates during the observation of actions, modulating the excitability of the motor cortex and the spinal cord when the movement is observed (Aziz-Zadeh et al. 2002; Baldissera et al. 2001; Fadiga et al. 1995). We have shown here that such processes are not just related to the execution phase, but are present also during the preparatory phase. The motor system facilitation we describe starts before movement onset, when subjects are simply expecting to see a movement in the future, and this matches the timing of the SE increase seen during active preparation for movement (Eichenberger and Ruegg 1984; Frank 1986; Mellah et al. 1990).

Additionally, we found that the motor system preparation for observing a movement involves muscles with different roles in the forthcoming movement, agonist, antagonist or synergist, and in all cases we found a SE increase, rather than an exclusive or stronger effect in the main agonist muscle involved in the observed movement. This muscle nonspecificity seems to be matched by processes of movement execution preparation (Hasbroucq et al. 1999). During actual MP, the increase in SE is part of a network process at which supraspinal centers seem to release movement by means of reducing inhibition on M1 (Davranche et al. 2007; Soto et al. 2010), allowing a "ready spinal cord" to trigger the muscle. The nonspecific increased SE found in our work may represent the marker of having the "ready spinal cord." What is interesting is that these subjects have "a ready spinal cord," despite the fact they are not going to execute any movement. When movement is executed, the cortical circuits will be modulated before the execution (Chen et al. 1998; Soto et al. 2010). In our protocol the movement was not going to be actually performed (and the subjects knew it), and that might be the reason such cortical modulation was absent.

The changes observed in our study seem a tonic adaptation of the spinal cord, maintained during a long period of time (the observed movement started between 3 and $4 \mathrm{~s}$ after the hand appearance). This process might be similar to the increase in the tonic motor unit (MU) discharge during MP (Mellah et al. 1990). The tonic discharge from slow MUs aims to increase the efficiency of the initially silent fast phasic MUs, which become activated at the triggering of movements (Mellah et al. 1990). The behavioral aim of this physiological substrate might center on environmental relations. We might be "spinally" ready to interact with someone in front of us if we expect their movements, but our supraspinal centers prepare and release the action only if needed.

We believe that our results present a new perspective on the study of MO which deserves to be explored deeper, as we have shown modulation in opposite directions depending on what the subject sees (hand or blank) and expects to see from a stationary hand. If we make the analogy between our simple passive observation task with a simple active reaction task (i.e., RT), in which the appearance of the hand after the blank was the warning (i.e., preparatory) signal, and the movement observed the response signal, it is possible that predictable and shorter foreperiods might have induced a different modulation of the CSE in our protocol (Hasbroucq et al. 1999). In fact, while CSE increases with the conditional probability of the response signal ( $100 \%$ in our experiments), it may decrease with its temporal predictability (with very restricted variation in our experiments) (Tandonnet et al. 2012). Thus we have avoided shorter

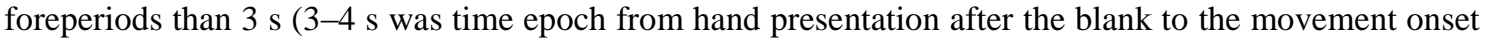
and stimulation). Additionally, the length of the foreperiods was not available to the subject, just to be sure we were away from time-windows compatible to processes related to "active motor preparation" like the Readiness Potential (Deecke et al. 1969), and to avoid an arousal increase in the early phase at hand pop-up after the blank. The view of the modulation of CSE during the preparation phase for MO might help gain considerable perspective on the meaning of CSE modulation during the observation of movement execution. 
It is also important to note how the spinal cord drives the behavior of our CS system when a stationary hand is visible, but, which the subject knows will not move compared with a neutral stimulus (BLACK). Our CS system was inhibited (or not facilitated). Clearly, then, what we describe here is an "inhibitory" mechanism related to the hand presence, and a facilitatory mechanism induced by the preparation of the CS tract for the movement we are going to observe (expectancy). Such processes might take place at the "slow" MUs of the spinal cord (Mellah et al. 1990). These slow MUs receive their excitatory input from the premotor cortex (PMc) and the supplementary motor area (Mellah et al. 1990; Tanji et al. 1988). Thus direct excitatory projections from secondary motor areas to the spinal cord, to motoneurons or its propiospinal circuits (Dum and Strick 1991, 2005; Lemon 2008), might modulate its excitability without a direct involvement of the M1, which is compatible with the spinal modulation observed in our study without modulation of corticocortical circuits involving M1 during pair-pulse exploration. Some of the secondary motor areas, like the PMc, receive projections either from the prefrontal cortex (Hanakawa 2011; Luppino et al. 2003) or from the dorsal visual system (Rizzolatti and Matelli 2003). The dorsal visual system is a functional network involving the posterior parietal cortex which receives input from the MT/V5 area, in turn modulated by V1 and other extrastriate visual areas (Rizzolatti and Matelli 2003). Thus the PMc might modulate the gain of the SE related to whether the hand is seen or not [since the PMc has a key role in integrating body parts (hand seen vs. hand not seen) to intended action (Hoshi and Tanji 2000)], and for this latter function the connections between prefrontal cortex and the PMc would be essential (Hanakawa 2011), allowing the transfer of cognitive information (the hand would move or would stay still) to the motor system.

In conclusion, motor system involvement during MO is bound not only to the movement phase, but to an earlier period at which the motor system becomes prepared to observe the forthcoming action, a process taking place at the spinal level, seen as an increase in its excitability.

\section{GRANTS}

This work was supported by Xunta de Galicia (Conselleria de Educación-2007/000140-0 and Dirección Xeral de I+D+i; 2010-2012), Spain. V. Robles-García and Y. Corral-Bergantiños received grants from the FPU-MECDAP2010-2774 and AP2010-2775 Spain.

\section{DISCLOSURES}

No conflicts of interest, financial or otherwise, are declared by the author(s).

\section{AUTHOR CONTRIBUTIONS}

Author contributions: P.A., K.L.G., A.O., and J.C. conception and design of research; P.A., V.R.-G., Y.C.-B., L.M.-M., A.O., and J.C. performed experiments; P.A., V.R.-G., and N.E. analyzed data; P.A., V.R.-G., N.E., Y.C.-B., L.M.-M., K.L.G., A.O., and J.C. interpreted results of experiments; P.A. and K.L.G. prepared figures; P.A. drafted manuscript; P.A., V.R.-G., N.E., Y.C.-B., L.M.-M., K.L.G., A.O., and J.C. edited and revised manuscript; P.A., V.R.-G., N.E., Y.C.-B., L.M.-M., K.L.G., A.O., and J.C. approved final version of manuscript. 


\section{REFERENCES}

Aoki H, Tsukahara R, Yabe K. Cortical and spinal motor excitability during the premovement EMG silent period. Brain Res 949: 178-187, 2002.

Aziz-Zadeh L, Maeda F, Zaidel E, Mazziotta J, Iacoboni M. Lateralization in motor facilitation during action observation: a TMS study. Exp Brain Res 144: 127-131, 2002.

Baldissera F, Cavallari P, Craighero L, Fadiga L. Modulation of spinal excitability during observation of hand actions in humans. Eur J Neurosci 13: 190-194, 2001.

Bonnet M, Decety J, Jeannerod M, Requin J. Mental simulation of an action modulates the excitability of spinal reflex pathways in man. Brain Res Cogn Brain Res 5: 221-228, 1997.

Cash RF, Ziemann U, Murray K, Thickbroom GW. Late cortical disinhi- bition in human motor cortex: a triple-pulse transcranial magnetic stimula- tion study. J Neurophysiol 103: 511-518, 2010.

Chen R, Yaseen Z, Cohen LG, Hallett M. Time course of corticospinal excitability in reaction time and self-paced movements. Ann Neurol 44: 317-325, 1998.

Conrad B, Benecke R, Goehmann M. Premovement silent period in fast movement initiation. Exp Brain Res 51: 310-313, 1983.

Darling WG, Cole KJ. Muscle activation patterns and kinetics of human index finger movements. J Neurophysiol 63: 1098-1108, 1990.

Davranche K, Tandonnet C, Burle B, Meynier C, Vidal F, Hasbroucq T. The dual nature of time preparation: neural activation and suppression revealed by transcranial magnetic stimulation of the motor cortex. Eur J Neurosci 25: 3766-3774, 2007.

Deecke L, Scheid P, Kornhuber HH. Distribution of readiness potential, pre-motion positivity, and motor potential of the human cerebral cortex preceding voluntary finger movements. Exp Brain Res 7: 158-168, 1969.

Di Lazzaro V, Oliviero A, Pilato F, Mazzone P, Insola A, Ranieri F, Tonali PA. Corticospinal volleys evoked by transcranial stimulation of the brain in conscious humans. Neurol Res 25: 143 $150,2003$.

Di Lazzaro V, Oliviero A, Profice P, Saturno E, Pilato F, Insola A, Mazzone P, Tonali P, Rothwell JC. Comparison of descending volleys evoked by transcranial magnetic and electric stimulation in conscious humans. Electroencephalogr Clin Neurophysiol 109: 397-401, 1998.

Di Lazzaro V, Rothwell JC, Oliviero A, Profice P, Insola A, Mazzone P, Tonali P. Intracortical origin of the short latency facilitation produced by pairs of threshold magnetic stimuli applied to human motor cortex. Exp Brain Res 129: 494-499, 1999.

di Pellegrino G, Fadiga L, Fogassi L, Gallese V, Rizzolatti G. Understand- ing motor events: a neurophysiological study. Exp Brain Res 91: 176-180, 1992.

Duclos Y, Schmied A, Burle B, Burnet H, Rossi-Durand C. Anticipatory changes in human motoneuron discharge patterns during motor preparation. J Physiol 586: 1017-1028, 2008.

Dum RP, Strick PL. Frontal lobe inputs to the digit representations of the motor areas on the lateral surface of the hemisphere. J Neurosci 25: 1375-1386, 2005.

Dum RP, Strick PL. The origin of corticospinal projections from the premotor areas in the frontal lobe. J Neurosci 11: 667-689, 1991.

Eichenberger A, Ruegg DG. Relation between the specific H reflex facilita- tion preceding a voluntary movement and movement parameters in man. J Physiol 347: 545-559, 1984.

Fadiga L, Fogassi L, Pavesi G, Rizzolatti G. Motor facilitation during action observation: a magnetic stimulation study. J Neurophysiol 73: 2608-2611, 1995.

Frank JS. Spinal motor preparation in humans. Electroencephalogr Clin Neurophysiol 63: 361-370, 1986. Hallett M. Transcranial magnetic stimulation and the human brain. Nature 406: 147-150, 2000. Hanakawa T. Rostral premotor cortex as a gateway between motor and cognitive networks. Neurosci Res 70: 144-154, 2011.

Hasbroucq T, Osman A, Possamai CA, Burle B, Carron S, Depy D, Latour S, Mouret I. Corticospinal inhibition reflects time but not event prepara- tion: neural mechanisms of preparation dissociated by transcranial magnetic stimulation. Acta Psychol (Amst) 101: 243-266, 1999

Hoshi E, Tanji J. Integration of target and body-part information in the premotor cortex when planning action. Nature 408: 466-470, 2000.

Hudson AL, Taylor JL, Gandevia SC, Butler JE. Coupling between me- chanical and neural behaviour in the human first dorsal interosseous muscle. J Physiol 587: 917-925, 2009.

Jeannerod M, Decety J. Mental motor imagery: a window into the represen- tational stages of action. Curr Opin Neurobiol 5: 727-732, 1995.

Lemon RN. Descending pathways in motor control. Annu Rev Neurosci 31: 195-218, 2008. 
Luppino G, Rozzi S, Calzavara R, Matelli M. Prefrontal and agranular cingulate projections to the dorsal premotor areas F2 and F7 in the macaque monkey. Eur J Neurosci 17: 559-578, 2003.

Martin PG, Hudson AL, Gandevia SC, Taylor JL. Reproducible measure- ment of human motoneuron excitability with magnetic stimulation of the corticospinal tract. J Neurophysiol 102: 606-613, 2009.

Maunsell JH, Ghose GM, Assad JA, McAdams CJ, Boudreau CE, Noerager BD. Visual response latencies of magnocellular and parvocellular LGN neurons in macaque monkeys. Vis Neurosci 16: 1-14, 1999.

Mellah S, Rispal-Padel L, Riviere G. Changes in excitability of motor units during preparation for movement. Exp Brain Res 82: 178-186, 1990.

Oldfield RC. The assessment and analysis of handedness: the Edinburgh inventory. Neuropsychologia 9: 97-113, 1971.

Requin J, Bonnet M, Somjen A. Is there a specificity in the supraspinal control of motor structures during preparation? In: Attention and Perfor- mance IV , edited by Dornic S. Hillsdale, NJ: Erlbaum, 1977.

Riedo R, Ruegg DG. Origin of the specific $\mathrm{H}$ reflex facilitation preceding a voluntary movement in man. J Physiol 397: 371-388, 1988.

Rizzolatti G, Matelli M. Two different streams form the dorsal visual system: anatomy and functions. Exp Brain Res 153: 146-157, 2003.

Rossini PM, Barker AT, Berardelli A, Caramia MD, Caruso G, Cracco RQ, Dimitrijevic MR, Hallett M, Katayama Y, Lucking $\mathrm{CH}$, et al. Non-invasive electrical and magnetic stimulation of the brain, spinal cord and roots: basic principles and procedures for routine clinical application. Report of an IFCN committee. Electroencephalogr Clin Neurophysiol 91: 79-92, 1994.

Soto O, Valls-Sole J, Kumru H. Paired-pulse transcranial magnetic stimula- tion during preparation for simple and choice reaction time tasks. J Neuro- physiol 104: 1392-1400, 2010.

Tandonnet C, Davranche K, Meynier C, Burle B, Vidal F, Hasbroucq T. How does temporal preparation speed up response implementation in choice tasks? Evidence for an early cortical activation. Psychophysiology 49: 252-260, 2012.

Tanji J, Okano K, Sato KC. Neuronal activity in cortical motor areas related to ipsilateral, contralateral, and bilateral digit movements of the monkey. J Neurophysiol 60: 325-343, 1988.

Thompson PD, Day BL, Rothwell JC, Dressler D, Maertens de Noordhout A, Marsden CD. Further observations on the facilitation of muscle re- sponses to cortical stimulation by voluntary contraction. Electroencephalogr Clin Neurophysiol 81: 397-402, 1991.

Ugawa Y, Rothwell JC, Day BL, Thompson PD, Marsden CD. Percutane- ous electrical stimulation of corticospinal pathways at the level of the pyramidal decussation in humans. Ann Neurol 29: 418-427, 1991.

Valls-Sole J, Pascual-Leone A, Wassermann EM, Hallett M. Human motor evoked responses to paired transcranial magnetic stimuli. Electroencepha- logr Clin Neurophysiol 85: 355-364, 1992.

van Elswijk G, Kleine BU, Overeem S, Stegeman DF. Expectancy induces dynamic modulation of corticospinal excitability. J Cogn Neurosci 19: 121-131, 2007.

Ziemann U, Rothwell JC, Ridding MC. Interaction between intracortical inhibition and facilitation in human motor cortex. J Physiol 496: 873-881, 1996.

Ziemann U, Tergau F, Wassermann EM, Wischer S, Hildebrandt J, Paulus W. Demonstration of facilitatory I wave interaction in the human motor cortex by paired transcranial magnetic stimulation. J Physiol 511: 181-190, 1998. 